jugular excision. The value of simple ligature of the internal jugular is prohlematical ; cases doing well after it would probably have recovered without interference with the vein. In dealing with sinus thrombosis it is always better to err on the side of radicalism.

Knowles Renshau.

\title{
NOSE.
}

Christie, N. A. (Welland, Ont.).--Nasal Diphtheria. "The Cun uliuı Prictitioner," January, 1912.

The patient, male, aged seventeen, was seen first on July 30, when diphtheria was diagnosed. The face was pallid, the tonsils and uvula covered with thick greyish membrane; from the nares issued a sanguinopurulent discharge, and cellulitis was so great about the angle of the right jaw that the upper and lower sets of teeth were not in line. The odour was characteristic of the disease.

During the first four days 18,000 units of antitoxin were alministered. On the sixth day the membrane commencel to loosen in the throat. On the seventh day profuse hæmorthuge occurred from the right nasial passage, and, on forcible blowing of the nose, an almost complete cast of the mucous membrane on that side was ejected. This membrane was very tough, and nearly one eighth of an inch thick. 'Io control the bleeding, a tampon soaked in solution of perehloride of iron wats inserted. 'This was removed on the following day, when a similar cast from the left nasal passage was blown out.

From this time there was marked daily improvement until the twelfth day, when the heart's action became weak and irregular. This was followed by pharyngeal and general paralysis with all the usual symptoms of this complicetion. Treatment wis by the ordinary methoils in such cases, including iron, strychnine and electricity, but the patient did not fully recover until four months from the date of the attack.

Price-Brown.

\section{THYROID GLAND.}

Farrant, R. Thyroid Action and Reaction. A Paper read before the Pathologieal Section of the Royal Society of Medicine on October 15,1912 .

'The author sairl that the thyroid secretion was absorbed by way of the thyroid veins. These veins corresponded in size to the thoracic duct and right lymphatic trunk ; they suarded the junction of the lymph with the venous circulation.

Certain toxins were alsorbed by the lymphatics and passed up through the terminal lymph-trunks to come into inmediate contact with the thyroid secretion. In these toxsmias a hyperplasia of the thyroid occurred without enlargement. This hyperplasia was illustrated by a series of slides taken from cases of infantile diarrhoea, diphtheria, measles, whooping-cough, broncho-pneumonia. So certain was this hyperplasia that in a given series the duration of the disease could be accurately arranged according to the degree of hyperplasia that had taken place. The reaction did not hend on the increased metabolism of febrile conditions, as it only occurred in certain diseases; for instance, infections with staphylococcus, 
streptococcus, Bucillus coli communis produced no reaction even when accompanied by high fever.

Experimental Toxiemin and Antitoric Action of the Thyroid.-The same hyperplasia could be proluced in guinea-pigs by injection with liphtheria toxin; the reaction was mitigated if at the same time thyroid was administered by the mouth.

He found that guinea-pigs injected daily with sublethal doses, and also with a single dose one and a half times the minimal lethal, lived longer if given thyroid by the mouth than those not so fed.

The serum of a thyroid-fed rabbit was found to be slightly antitoxic to diphtheria toxin; the smallest dose of serum given in this series wats .25 c.e.; it protected a guinea-pig of $270 \mathrm{~g}$. from one and a half times the minimal lethal dose of diphtheria toxin. All the controls died.

Diphtheria antitoxin, when fed to a normal rabbit, produced symptoms similar to those of hyperthyroidism; the rabbit died in sixteen days, having lost 31 per cent. of its weight.

Rabbits fed on normal horse-serum developed no such symptoms and put on weight. Diphtheria antitoxin fed to thyrodectomised animals produced no ill-effect.

Observations on Antituxin.-A series of sections of horse thyroids was shown that had been subjected to the effect of diphtheria antitoxin for one year. This series was arranged in the orler of hyperplasia: the first section showed rows of cells arranged around small vesicles containing a little colloid, the last showed a colloidal thyroid with the cells squeezed together into rows between the larger vesicles. The direct transformation of the cells into colloid was seen in these sections. The antitoxic value of the serum was in the reverse order of the hyperplasia, the most hyperplastic thyroid corresponding to the lowest antitoxic value.

Relation between the Thyroid Hyperplasia, the Antituric Value, and their Iodine Valme.-Hyperplasia varied inversely with the iodine content - Marine and Williams. The thyroid with low antitoxic value would thus show a low iodine value. From estimations on the iodine value of diphtheria antitoxin, he found that the antitoxic value varied with the iodiue value. Normal horse-serum was found to contain but the slightest trace of iodine. Marine and Williams had showed that the plyssiological value of the thyroid varied with its iodine value.

Mr. Farrant put these results together: Certain toxins simulated the thyroid into a condition of hyperplasia, during this change it seemed probable that the ioline-containing substance was poured out into the circulation, for the serum of an immunised horse contained an excess of ionline, while the thyroid was hyperplastic. He assumed from the experiments of Marine and Williams that the thyroid itself became deficient in ionline at the same time. During the reversion back to the colloild, the ioline was once more taken up by the thyroid and it changed back to the colloid gland ; the rate of change was directly proportional to the amount of iodine present.

Some close relationship was thus indicated between the thyroid function and the development of certain antitoxin: He suggested that perhaps the hyperplasia observed in these toxwmias arose from the attempt to form antitoxin.

The Formution of 'Thyroid Tumomis: (a) Normal to Hyperplasia.The toxins might be divided into the exogenous, producing enlenic hyperplasia, the endogenous, producing sporddic hyperplasia. In both if the toxin was removed early no enlargement took place. 'The similarity between the hyperplasia produced by measles and broncho-pneumonia of 
ten days' duration and exophthalmic goitre was shown; the only difference was that the early had not produced an enlargement whilst the late had produced an even enlargement of the thyroid.

(b) Hyperplasia to Colloid.-The reversion from hyperplasia to colloid was shown by a slide of early broncho-pneumonia and one dead three months after recovery. The similarity between the colloid reversion of an early and a late hyperplasia was shown. This deposit of colloid would be most marked in those portions of the thyroid that had been most hyperplastic; this would leal to the formation of adenomata; the rest of the gland might be in any degree of hyperplasia. During this involution degenerative changes were liable to occur, producingr single or miltiple cysts. The involution might go on to complete fibrosis.

Hyperplasia without thyorid enlargement.

Hyperplasia with various degrees of enlargement.

Adenomata of involution.

Cysts and cyst-adenomata of degeneration.

Fibrosis.

The formation of thyroid tumours in cretins.- Seventy five per cent of cretins had at one time or other thyroid tumours; the cycle of changes from hyperplasia to atrophy occurred in two to three years, when symptoms of cretinism developed. Two facts accounted for this rapidity of change. The toxin circulating in the blood of the fotus would be relatively large in amount as it would correspond to the toxicity of the mother's blood. Secondly, the normal thyroid of a foetus was already in a condition corresponding to that of hyperplasia, and owing to the delicate condition of the fuetal organs one would expect the changes from hyperplasia to atrophy to occur rapidly.

Anthor's Alstract.

\section{REVIEWS.}

Die Laryngealen Erscheinungen bei multipler Sklerose des Gehirns und Kuchenmarks (The Laryngeal Manifestations in Multiple Sclerosis of the Brain and Spinal Cord). By Privatdozent Ir. L. RÉтн (Vienna). Pp. 148. Viemna: Josef Safar, 1907.

The relations of "special" symptoms to general diseases are always of the greatest interest to the specialist who respects his special department, and who is too conscientious to run the risk of overlooking the real nature of the more olsscure of the conditions affecting the parts of the body with which he is particularly, or, it may be, exclusively occupied. It is when the special symptoms are the first to show themselves, and the signs of the general disease are not developed sufficiently for its nature to be recognised by the non-expert, that the interest and the importance of their correct interpretation are most marked. Occasionally disseminated sclerosis is ushered in by laryngeal symptoms, such as hoarseness (p. 27), but this is unusual. It cannot, indeed, be said that laryngeal paralysis is a common occurrence in disseminated sclerosis, but fluctuating degrees of disturbance of mobility of the cords are fairly frequent. Rethi in his monograph analyses the various laryngeal and vocal disturhances very thoroughly by an exhaustive study of the cases published by other anthors, enhanced by his observations on his own cases. In general it may be accepted that the various disturbances in the action of the laryngeal muscles are phenomena of weakness rather than of paralysis. 'The work is strictly objective and devoid of anything in the way of padding. Its study will provide the laryngologist with the means of 